\title{
Profilaxis primaria de várices esofágicas en pediatría
}

\author{
JUAN CRISTÓBAL GANAA. ${ }^{1}$ \\ 1. División de Pediatría, Unidad de Gastroenterología, Hepatología y Nutrición. \\ Pontificia Universidad Católica de Chile.
}

\begin{abstract}
Primary prophylactic treatment of esophageal varices in pediatrics

Children with chronic liver disease or portal vein thrombosis frequently develop portal hypertension. The main complication of the portal hypertension are the development of esophageal varices who are at risk of variceal bleeding, which is associated with significant morbidity and mortality. Guidelines for adults with portal hypertension recommend performing esophagogastroduodenoscopy to identify those with varices that may benefit from prophylactic therapy, however there are no consensus in the pediatric age. This revision includes the incidence, diagnostic and the evidence for primary prophylactic treatment of the esophageal varices in children. (Key words: Esophageal varices, portal hypertension, primary prophylaxis, chronic liver disease).

Rev Chil Pediatr 2011; 82 (5): 381-387
\end{abstract}

\section{RESUMEN}

Pacientes pediátricos con daño hepático crónico o con trombosis de la vena porta frecuentemente desarrollan hipertensión portal. La principal complicación de la hipertensión portal es el desarrollo de várices esofágicas que conlleva riesgo de hemorragia variceal, la cual se asocia a significativa morbimortalidad. Las guías clínicas para adultos recomiendan realizar, en pacientes cirróticos, una endoscopia como screening para el diagnóstico de várices esofágicas y su tratamiento profiláctico, sin embargo, en pacientes pediátricos no existe consenso. En esta revisión se actualiza la incidencia, diagnóstico y evidencia del tratamiento profiláctico primario de várices esofágicas en pediatría.

(Palabras clave: Várices esofágicas, hipertensión portal, profilaxis primaria, daño hepático crónico).

Rev Chil Pediatr 2011; 82 (5): 381-387

\section{Introducción}

La historia natural de la enfermedad hepática crónica implica un aumento de la presión portal debido al incremento de la resistencia del flujo hepático producto de la distorsión hepática por tejido fibroso y nódulos regenerativos. Además de esta resistencia estructural, existe una vasoconstricción intrahepática activa secundaria a una disminución endógena de

Trabajo recibido el 29 de septiembre de 2011, aceptado el 15 de octubre de 2011.

Correspondencia a:

Juan Cristóbal Gana A.

E-mail: jcgana@gmail.com 
la producción de oxido nítrico ${ }^{1,2}$ que incrementa en un 20 a $30 \%$ la resistencia intrahepática ${ }^{3}$. Este aumento en la presión portal es el que desencadena la formación de colaterales portosistémicas, lo que implica un aumento del riesgo de complicaciones, incluidas la hemorragia por várices esofágicas y gástricas.

Los objetivos de esta revisión son actualizar la evidencia disponible en la literatura en cuanto a la incidencia, diagnóstico y tratamiento profiláctico primario de várices esofágicas en pediatría.

\section{Várices esofágicas en hipertensión portal en adultos}

La prevalencia de cirrosis en adultos es de 0,4 a $1,1 \%$ en países desarrollados ${ }^{4,5}$. Las várices gastroesofágicas se encuentran aproximadamente en el $50 \%$ de los pacientes cirróticos. Su presencia de correlaciona con la severidad de la enfermedad hepática; así por ejemplo, solo un $40 \%$ de los pacientes Child A presentan várices, a diferencia de pacientes Child $\mathrm{C}$ en que la incidencia alcanza un $85 \%{ }^{6}$. Adultos cirróticos sin várices esofágicas tienen un riesgo de desarrollar várices esofágicas en un $5 \%$ anual $^{7}$, siendo el principal predictor de desarrollo la gradiente de presión venosa hepática $^{8}$. Pacientes con várices pequeñas progresan a grandes a una incidencia anual entre un $5-10 \%{ }^{9}$.

Una vez que las várices esofágicas se desarrollan, el riesgo de hemorragia varía entre un $20-35 \%$ a los 2 años ${ }^{10,11}$. Los predictores mas importantes de hemorragia incluyen la cirrosis descompensada y la presencia endoscópica de várices grandes y signos rojos ${ }^{12}$ (figura 1). Aunque la hemorragia por várices esofágicas cede espontáneamente en hasta un $40 \%$, se asocia a una mortalidad de hasta un $20 \%$ a las 6 semanas $^{13-15}$.

\section{Várices esofágicas en hipertensión portal en pediatría}

Aunque no hay estudios de prevalencia de várices esofágicas en pediatría, estudios en centros de referencia hepatológica sugieren que mas de un $50 \%$ de niños con cirrosis hepática presentan várices esofágicas ${ }^{16,17}$.

La hemorragia por várices esofágicas ocurre frecuentemente en niños con cirrosis o trombosis de la vena porta ${ }^{18-25}$. En niños con atresia de las vías biliares, la incidencia de hemorragia por várices esofágicas ocurre en un 17 a $29 \%$ en los primeros 5 a 10 años $^{20-22}$ y en hasta un $50 \%$ en niños que sobreviven mas de 10 años en quienes no han requerido un trasplante hepático ${ }^{23}$. Recientemente se publicó un artículo que reporta 125 niños con atresia de vías biliares con signos de hipertensión portal o previa historia de hemorragia digestiva sometidos a endoscopía digestiva alta, en una media de 13 meses (rango 1-14 años) y se identificaron $88(70 \%)$ pacientes con várices esofágicas $^{26}$.

Entre 50 niños con várices esofágicas, principalmente secundario a cirrosis, seguidos en forma prospectiva durante 4,5 años y sin tratamiento profiláctico, un $42 \%$ presentó hemorragia gastrointestinal alta ${ }^{19}$. En niños con trombosis de la vena porta hasta en un $50 \%$
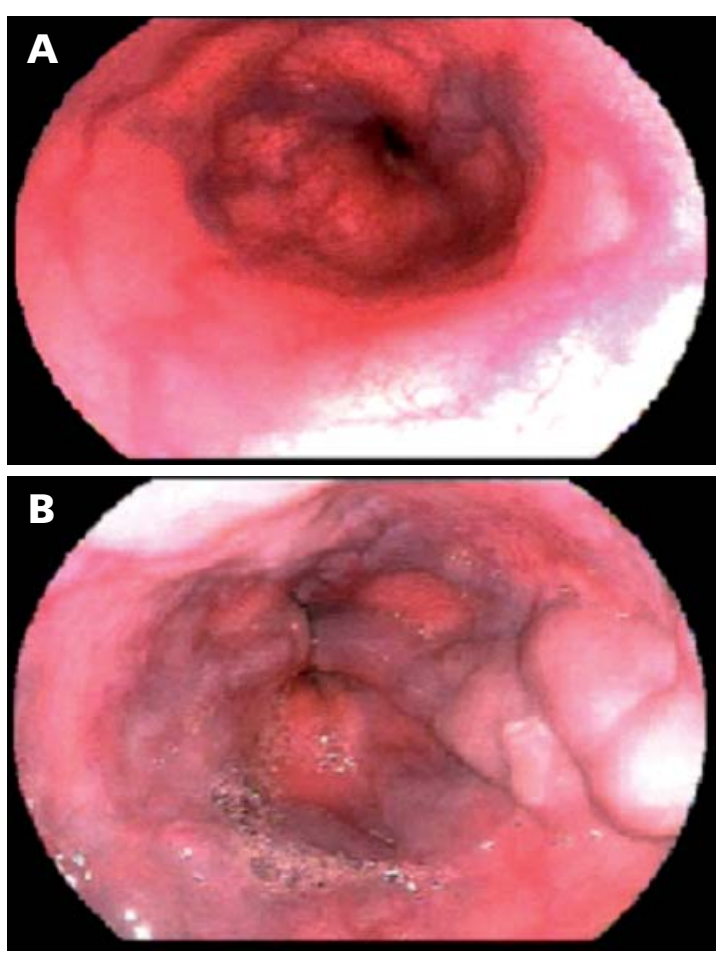

Figura 1. Varices esofágicas chicas (panel A) y grandes (panel B). 
han sufrido una hemorragia digestiva alta a los 16 años de edad ${ }^{18}$.

A diferencia de los adultos, hay pocos datos disponibles para estimar el riesgo de hemorragia variceal. En pacientes con atresia de vías biliares la presencia de signos rojos y várices gástricas resultaron ser los parámetros endoscópicos mas importantes de riesgo de hemorragia $^{27}$. En cuanto a factores de riesgo no endoscópicos, solo hay un trabajo caso-control el cual describe a través de una regla de predicción clínica (que incluye el número de plaquetas, la albúmina y el tamaño del bazo) el riesgo de hemorragia por várices esofágicas ${ }^{28}$. Probablemente la combinación de la regla de predicción clínica junto a los hallazgos endoscópicos variceales sean buenos predictores de hemorragia, estudio que está en proceso validación.

La mortalidad por hemorragia de várices esofágicas ha sido descrita entre un 2,5 a $20 \%$ de niños con hipertensión portal $1^{19,21,24,25}$.

\section{Diagnóstico}

El método usado actualmente para diagnosticar várices esofágicas es la endoscopía digestiva alta. En adultos, la recomendación actual es la realización de endoscopía como screening a todos los pacientes cirróticos. Sin embargo, debido a la falta de evidencia de terapia profiláctica en pediatría, aún no hay consenso en este grupo etáreo. En una reciente encuesta realizada a Gastroenterólogos Pediátricos en Canadá, un $70 \%$ considera la realización de screening con endoscopía para várices esofágicas en niños con evidencia de cirrosis o hipertensión portal ${ }^{29}$. Interesantemente, la mayoría de los pacientes con hipertensión portal y sus familias demostraron interés por la realización de endoscopía incluso si se ofrece solo como herramienta diagnóstica y no terapéutica y así conocer su riesgo de hemorragia.

Por otro lado, la endoscopía en un método invasivo, caro, asociado a posibles complicaciones y en niños requiere sedación o incluso en algunos centros anestesia. Por esta razón, se han intentado desarrollar métodos no invasivos de diagnóstico de várices esofágicas.
Estos podrían reemplazar el uso de la endoscopía, o bien ser utilizados para identificar a los niños con mayor riesgo de várices, como screening previo a la endoscopía. La Asociación Americana de Estudios de Enfermedades Hepáticas (AASLD) y el Colegio Americano de Gastroenterología (ACG) reconocen esta área como una de las más importantes en la investigación relacionada con la hipertensión portal ${ }^{6}$. Debido a la amplia variedades de tests para diagnóstico de várices, que incluyen diversos tests de laboratorio (como recuento plaquetario y albúmina) e imágenes (cápsula endoscópica, elastografía, resonancia magnética y otros), estamos actualmente finalizando cinco revisiones sistemáticas para la colaboración Cochrane que esperamos ayuden en la toma de decisiones futuras ${ }^{30-34}$.

Existen pocos estudios en niños que evalúen tests no invasivos para el diagnóstico de várices esofágicas ${ }^{35-37}$. Un estudio retrospectivo de 51 pacientes con hipertensión portal secundario a enfermedad hepática o trombosis de la vena porta, describió que los mejores parámetros no-invasivos para el diagnóstico de várices esofágicas fueron la relación entre el número de plaquetas y el tamaño del bazo (en valor z) y una regla de predicción clínica que incluye el recuento plaquetario, el tamaño del bazo y el valor de la albúmina, con áreas bajo la curva ROC de 0,91 y 0,93 , respectivamente. Estos resultados sugieren que a través de métodos no invasivos, se podría seleccionar pacientes para screening de várices con endoscopía. Estos resultados fueron validados recientemente en un estudio prospectivo multicéntrico ${ }^{38}$.

\section{Profilaxis primaria}

El tratamiento con $\beta$-bloqueadores y ligadura endoscópica han demostrado ser eficientes en la prevención de hemorragia por várices esofágicas en adultos ${ }^{39-42}$. A continuación se describe la evidencia publicada en niños.

\section{1) B-bloqueadores}

Se han publicado seis trabajos en que reportan experiencia clínica con uso de $\beta$-bloquea- 
Tabla 1. Estudios de profilaxis primaria en pediatría

\begin{tabular}{|llccc|}
\hline & Diseño & Número & Seguimiento (años) & Hemorragia \\
\hline$\beta$-bloqueadores & & 17 & 3 & 35 \\
Shashidhar (45) & Series de casos & 45 & 5 & 16 \\
Ozsoylu (46) & Series de casos & 10 & 5,2 & 10 \\
Erkan (48) & Series de casos & & No disponible & 0 \\
Ligadura & & 4 & 2 & 10 \\
Cano (50) & Series de casos & 9 & 1,5 & 0 \\
Sasaki (51) & Series de casos & 37 & & 0 \\
Celinska-Cedro (52) & Series de casos & & 10 & 0 \\
Escleroterapia & & 2 & 2,5 & 42 \\
Paquet (53) & Series de casos & 17 & 2,4 & 6 \\
Howard (54) & Series de casos & 26 & 4,5 & 8 \\
Maksoud (55) & Series de casos & 100 & 0,7 & \\
Goncalves (19) & Randomizado controlado & 13 & & \\
Duché (56) & Series de casos & & & 0 \\
\hline
\end{tabular}

dores no selectivos en niños con hipertensión portal $^{43-48}$, algunas incluyen terapia profiláctica primaria (tabla 1). En total conforman 131 pacientes con distintas etiologías de daño hepático crónico. No hay estudios randomizados controlados, solo existen reportes de series clínicas que evalúan el uso de $\beta$-bloqueadores como profilaxis primaria con períodos de seguimiento de hasta 5 años, con una hemorragia reportada entre un 10 y $35 \%$.

La mayoría de los trabajos reportados en pediatría incluyen la reducción de la frecuencia cardíaca en un $25 \%$ como respuesta biológica óptima al uso de $\beta$-bloqueadores. Sin embargo, ninguno de estos estudios ha demostrado que este cambio se traduzca en una disminución en la gradiente de presión venosa hepática o que este punto de corte se correlacione con una mejoría clínica. La dosis de propanolol varía en los estudios desde 1 a $8 \mathrm{mg} / \mathrm{kg} /$ día.

La eficaciaterapéutica de los $\beta$-bloqueadores no se puede determinar de los estudios publicados en pacientes pediátricos, especialmente debido a la falta de data de grupos control. Aunque no se han descrito serias complicaciones en pacientes con hemorragia por várices, en este grupo etáreo debe tenerse especial cuidado ya que los niños utilizan el aumento de su frecuencia cardíaca como principal meca- nismo de compensación hemodinámica frente a la hipovolemia. Son necesarios mayores estudios antes de su recomendación profiláctica universal.

\section{2) Terapia endoscópica}

La ligadura variceal endoscópica (figura 2) ha reemplazado a la escleroterapia como tratamiento de elección para la profilaxis primaria y secundaria. Se han reportado algunos reportes no randomizados sobre ligadura endoscópica para profilaxis primaria para várices esofágicas en niños (tabla 1). Estos han demostrado

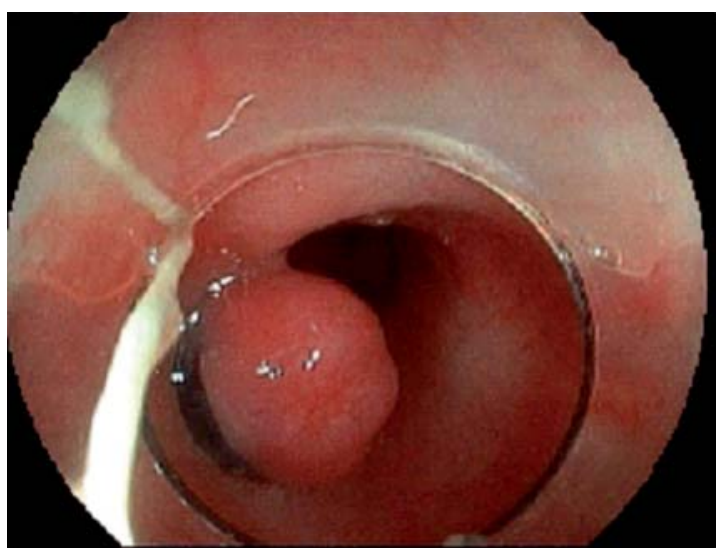

Figura 2. Ligadura endoscópica de várice esofágicas. 
que la ligadura es bien tolerada en niños con baja tasa de hemorragia y sin mayores complicaciones. Solo hay un trabajo randomizado controlado sobre terapia endoscópica para profilaxis primaria ${ }^{19}$. Este estudio comparó niños con terapia de escleroterapia para sus várices versus control sin tratamiento y demostró una reducción de un $50 \%$ en el riesgo de hemorragia en un período de 4 años, pero sin efecto en mortalidad. Solo existe un estudio randomizado controlado pediátrico en que se compara terapia endoscópica de ligadura versus escleroterapia, pero realizado como profilaxis secundaria, el cual demostró una diferencia significativa en la tasa de hemorragia a favor de la ligadura ( $25 v s 4 \%)^{49}$. En encuesta a gastroenterólogos y hepatólogos en Canadá, la mayoría opta por realizar profilaxis primaria, de preferencia la ligadura endoscópica ${ }^{29}$.

Aunque la incorporación de nuevos endoscopios pediátricos de menor calibre ha logrado realizar ligaduras variceales en pre-escolares, aun no es posible en lactantes debido al tamaño, por lo que la única terapia posible endoscópica en este tipo de pacientes es la escleroterapia.

\section{Conclusiones}

Las várices esofágicas son una complicación frecuente en Pediatría en pacientes con atresia de vías biliares y trombosis de la vena porta y presentan una morbimortalidad considerable. Existen nuevos métodos no-invasivos para el diagnóstico de várices esofágicas que podrían seleccionar a los pacientes para la realización ulterior de endoscopía digestiva.

Debido a la escasa evidencia sobre terapia profiláctica para várices esofágicas, aún no hay consenso pediátrico sobre su uso. Sin embargo, en encuestas a gastroenterólogos, la mayoría opta por realizar profilaxis primaria, de preferencia la ligadura endoscópica. No obstante, cada paciente debe ser individualizado de acuerdo a su edad, riesgo endoscópico de hemorragia y según disponibilidad de acceso a un centro especializado al momento de la toma de decisiones, que debe ser tomada en conjunto con el paciente y su familia.

\section{Agradecimientos}

Se agradece especialmente a Margarita Mena por su contribución en el soporte y gráfica de este artículo.

\section{Referencias}

1.- Gupta TK, Toruner M, Chung MK, Groszmann RJ: Endothelial dysfunction and decreased production of nitric oxide in the intrahepatic microcirculation of cirrhotic rats. Hepatology 1998; 28 (4): 926-31.

2.- Wiest R, Groszmann RJ: Nitric oxide and portal hypertension: its role in the regulation of intrahepatic and splanchnic vascular resistance. Semin Liver Dis 1999; 19 (4): 411-26.

3.- Bhathal PS, Grossman HJ: Reduction of the increased portal vascular resistance of the isolated perfused cirrhotic rat liver by vasodilators. J Hepatol 1985; 1 (4): 325-37.

4.- Quinn PG, Johnston DE: Detection of chronic liver disease: costs and benefits. Gastroenterologist 1997; 5 (1): 58-77.

5.- Bellentani S, Tiribelli C, Saccoccio G, et al: Prevalence of chronic liver disease in the general population of northern Italy: the Dionysos Study. Hepatology 1994; 20 (6): 1442-9.

6.- García-Tsao G, Sanyal AJ, Grace ND, Carey W: Prevention and management of gastroesophageal varices and variceal hemorrhage in cirrhosis. Hepatology 2007; 46 (3): 922-38.

7.- Garceau AJ, Chalmers TC: The natural history of cirrhosis. I. Survival with esophageal varices. N Engl J Med 1963; 268: 469-73.

8.- Groszmann RJ, García-Tsao G, Bosch J, et al: Betablockers to prevent gastroesophageal varices in patients with cirrhosis. N Engl J Med 2005; 353 (21): 2254-61.

9.- Merli M, Nicolini G, Angeloni S, et al: Incidence and natural history of small esophageal varices in cirrhotic patients. J Hepatol 2003; 38 (3): 266-72.

10.- Prediction of the first variceal hemorrhage in patients with cirrhosis of the liver and esophageal varices. A prospective multicenter study. The North Italian Endoscopic Club for the Study and Treatment of Esophageal Varices. N Engl J Med 1988; 319 (15): 983-9.

11.- Gores GJ, Wiesner RH, Dickson ER, Zinsmeister AR, Jorgensen RA, Langworthy A: Prospective evaluation of esophageal varices in primary biliary cirrhosis: development, natural history, and influence on survival. Gastroenterology 1989; 96 (6): 1552-9. 
12.- Prediction of the first variceal hemorrhage in patients with cirrhosis of the liver and esophageal varices. A prospective multicenter study. The North Italian Endoscopic Club for the Study and Treatment of Esophageal Varices. N Engl J Med 1988; 319 (15): 983-9.

13.- El-Serag HB, Everhart JE: Improved survival after variceal hemorrhage over an 11-year period in the Department of Veterans Affairs. Am J Gastroenterol 2000; 95 (12): 3566-73.

14.- D’Amico $G$, de Franchis $R$ : Upper digestive bleeding in cirrhosis. Post-therapeutic outcome and prognostic indicators. Hepatology 2003; 38 (3): 599-612.

15.- Carbonell N, Pauwels A, Serfaty L, Fourdan O, Levy $V G$, Poupon R: Improved survival after variceal bleeding in patients with cirrhosis over the past two decades. Hepatology 2004; 40 (3): 652-9.

16.- Sokal EM, Van HN, Van OL, Otte JB, Buts JP: Upper gastro-intestinal tract bleeding in cirrhotic children candidates for liver transplantation. Eur J Pediatr 1992; 151 (5): 326-8.

17.- Stringer MD, Howard ER, Mowat AP: Endoscopic sclerotherapy in the management of esophageal varices in 61 children with biliary atresia. J Pediatr Surg 1989; 24 (5): 438-42.

18.- Lykavieris P, Gauthier F, Hadchouel P, Duche M, Bernard O: Risk of gastrointestinal bleeding during adolescence and early adulthood in children with portal vein obstruction. J Pediatr 2000; 136 (6): 805-8.

19.- Goncalves ME, Cardoso SR, Maksoud JG: Prophylactic sclerotherapy in children with esophageal varices: longterm results of a controlled prospective randomized trial. J Pediatr Surg 2000; 35 (3): 401-5.

20.- Miga D, Sokol RJ, Mackenzie T, Narkewicz MR, Smith $D$, Karrer FM: Survival after first esophageal variceal hemorrhage in patients with biliary atresia. J Pediatr 2001; 139 (2): 291-6.

21.- van Heurn LW, Saing H, Tam PK: Portoenterostomy for biliary atresia: Long-term survival and prognosis after esophageal variceal bleeding. J Pediatr Surg 2004; 39 (1): 6-9.

22.- Kobayashi A, Itabashi F, Ohbe Y: Long-term prognosis in biliary atresia after hepatic portoenterostomy: analysis of 35 patients who survived beyond 5 years of age. J Pediatr 1984; 105 (2): 243-6.

23.- Toyosaka A, Okamoto E, Okasora T, Nose K, Tomimoto $Y$ : Outcome of 21 patients with biliary atresia living more than 10 years. J Pediatr Surg 1993; 28 (11): 1498501.

24.- Mitra SK, Kumar V, Datta DV, et al: Extrahepatic portal hypertension: a review of 70 cases. J Pediatr Surg 1978;
13 (1): 51-7.

25.- Webb LJ, Sherlock $S$ : The aetiology, presentation and natural history of extra-hepatic portal venous obstruction. Q J Med 1979; 48 (192): 627-39.

26.- Duche M, Ducot B, Tournay E, et al: Prognostic value of endoscopy in children with biliary atresia at risk for early development of varices and bleeding. Gastroenterology 2010; 139 (6): 1952-60.

27.- Duche $M$, Ducot B, Tournay E, et al: Prognostic value of endoscopy in children with biliary atresia at risk for early development of varices and bleeding. Gastroenterology 2010; 139 (6): 1952-60.

28.- Gana JC, Turner D, Avitzur Y, Ling SC: Prediction of esophageal variceal bleeding in children [Abstract]. Gastroenterology 2009; 136 (5): A-825.

29.- Gana JC, Valentino PL, Morinville V, O’Connor C, Ling SC: Variation in care for children with esophageal varices: a study of physicians', patients', and families' approaches and attitudes. J Pediatr Gastroenterol Nutr 2011; 52 (6): 751-5.

30.- Gana JC, Turner D, Yap J, Adams-Webber T, Rashkovan $N$, Ling SC: Magnetic resonance imaging, computer tomography scan, and oesophagography for the diagnosis of oesophageal varices in patients with chronic liver disease or portal vein thrombosis (Protocol). Cochrane Database of Systematic Reviews 2010; (10): Art. No.: CD008763. DOI: 10.1002/14651858.CD008763.

31.- Gana JC, Turner D, Yap J, Adams-Webber T, Rashkovan $N$, Ling SC: Platelet count, spleen length, and platelet count/spleen length ratio for the diagnosis of oesophageal varices in patients with chronic liver disease or portal vein thrombosis (Protocol). Cochrane Database of Systematic Reviews 2010; (10): Art. No.: CD008759. DOI: 10.1002/14651858.CD008759.

32.- Gana JC, Turner D, Yap J, Adams-Webber T, Rashkovan $N$, Ling SC: Transient ultrasound elastography and magnetic resonance elastography for the diagnosis of oesophageal varices in patients with chronic liver disease or portal vein thrombosis (Protocol). Cochrane Database of Systematic Reviews 2010; (10): Issue 10. Art. No.: CD008761. DOI: 10.1002/14651858.CD008761.

33.- Gana JC, Turner D, Yap J, Adams-Webber T, Rashkovan $N$, Ling SC: Capsule endoscopy for the diagnosis of oesophageal varices in patients with chronic liver disease or portal vein thrombosis (Protocol). Cochrane Database of Systematic Reviews 2010; (10): Art. No.: CD008760. DOI:10.1002/14651858.CD008760.

34.- Gana JC, Turner D, Yap J, Adams-Webber T, Rashkovan $N$, Ling SC: Non-invasive test of liver fibrosis for the diagnosis of oesophageal varices in patients with 
chronic liver disease or portal vein thrombosis (Protocol). Cochrane Database of Systematic Reviews 2010; (10): Art. No.: CD008764. DOI: 10.1002/14651858. CD008764.

35.- Gana JC, Turner D, Roberts EA, Ling SC: Derivation of a clinical prediction rule for the noninvasive diagnosis of varices in children. J Pediatr Gastroenterol Nutr 2010; 50 (2): 188-93.

36.- Fagundes ED, Ferreira AR, Roquete $M L$, et al: Clinical and laboratory predictors of esophageal varices in children and adolescents with portal hypertension syndrome. J Pediatr Gastroenterol Nutr 2008; 46 (2): 178-83.

37.- Chang HK, Park YJ, Koh H, et al: Hepatic fibrosis scan for liver stiffness score measurement: a useful preendoscopic screening test for the detection of varices in postoperative patients with biliary atresia. J Pediatr Gastroenterol Nutr 2009; 49 (3): 323-8.

38.- Gana JC, Turner D, Mieli-Vergani G, et al: A Clinical Prediction Rule and Platelet Count Predict Esophageal Varices in Children. Gastroenterology 2011 Sep 14.

39.- Khuroo MS, Khuroo NS, Farahat KL, Khuroo YS, Sofi AA, Dahab ST: Meta-analysis: endoscopic variceal ligation for primary prophylaxis of oesophageal variceal bleeding. Aliment Pharmacol Ther 2005; 21 (4): 34761.

40.- García-Pagan JC, Bosch J: Endoscopic band ligation in the treatment of portal hypertension. Nat Clin Pract Gastroenterol Hepatol 2005; 2 (11): 526-35.

41.- Triantos CK, Burroughs AK: Prevention of the development of varices and first portal hypertensive bleeding episode. Best Pract Res Clin Gastroenterol 2007; 21 (1): 31-42.

42.- D’Amico G, Pagliaro L, Bosch J: Pharmacological treatment of portal hypertension: an evidence-based approach. Semin Liver Dis 1999; 19 (4): 475-505.

43.- Ozsoylu S, Kocak N, Yuce A: Propranolol therapy for portal hypertension in children. J Pediatr 1985; 106 (2): 317-21.

44.- de Kolster CC, Rapa de HM, Carvajal A, Castro J, Callegari $C$, Kolster $J$ : [Propranolol in children and adolescents with portal hypertension: its dosage and the clinical, cardiovascular and biochemical effects]. G E N 1992; 46 (3): 199-207.

45.- Shashidhar H, Langhans N, Grand RJ: Propranolol in prevention of portal hypertensive hemorrhage in chil- dren: a pilot study. J Pediatr Gastroenterol Nutr 1999; 29 (1): 12-7.

46.- Ozsoylu S, Kocak N, Demir H, Yuce A, Gurakan F, Ozen $H$ : Propranolol for primary and secondary prophylaxis of variceal bleeding in children with cirrhosis. Turk $\mathrm{J}$ Pediatr 2000; 42 (1): 31-3.

47.- Sokucu S, Suoglu OD, Elkabes B, Saner G: Long-term outcome after sclerotherapy with or without a betablocker for variceal bleeding in children. Pediatr Int 2003; 45 (4): 388-94.

48.- Erkan T, Cullu F, Kutlu T, et al: Management of portal hypertension in children: a retrospective study with long-term follow-up. Acta Gastroenterol Belg 2003; 66 (3): 213-7.

49.- Zargar SA, Javid G, Khan BA, et al: Endoscopic ligation compared with sclerotherapy for bleeding esophageal varices in children with extrahepatic portal venous obstruction. Hepatology 2002; 36 (3): 666-72.

50.- Cano I, Urruzuno P, Medina E, et al: Treatment of esophageal varices by endoscopic ligation in children. Eur J Pediatr Surg 1995; 5 (5): 299-302.

51.- Sasaki T, Hasegawa T, Nakajima K, et al: Endoscopic variceal ligation in the management of gastroesophageal varices in postoperative biliary atresia. J Pediatr Surg 1998; 33 (11): 1628-32.

52.- Celinska-Cedro D, Teisseyre $M$, Woynarowski M, Socha P, Socha J, Ryzko J: Endoscopic ligation of esophageal varices for prophylaxis of first bleeding in children and adolescents with portal hypertension: preliminary results of a prospective study. J Pediatr Surg 2003; 38 (7): 1008-11.

53.- Paquet KJ: Ten years experience with paravariceal injection sclerotherapy of esophageal varices in children. J Pediatr Surg 1985; 20 (2): 109-12.

54.- Howard ER, Stringer MD, Mowat AP: Assessment of injection sclerotherapy in the management of 152 children with oesophageal varices. Br J Surg 1988; 75 (5): 404-8.

55.- Maksoud JG, Goncalves ME, Porta G, Miura I, Velhote $M C$ : The endoscopic and surgical management of portal hypertension in children: analysis of 123 cases. J Pediatr Surg 1991; 26 (2): 178-81.

56.- Duche M, Habes D, Roulleau P, Haas V, Jacquemin E, Bernard O: Prophylactic endoscopic sclerotherapy of large esophagogastric varices in infants with biliary atresia. Gastrointest Endosc 2008; 67 (4): 732-7. 